\title{
Open Versus Minimally Invasive Resection of Gastric GIST: A Multi-Institutional Analysis of Short- and Long-Term Outcomes: A Reply
}

\author{
Danielle A. Bischof, MD, MHS, FRCSC, FACS ${ }^{1}$ and Timothy M. Pawlik, MD, MPH, PhD, FACS ${ }^{2}$ \\ ${ }^{1}$ Department of Surgery, Mount Sinai Hospital, Toronto, ON, Canada; ${ }^{2}$ The Ohio State University Wexner Medical Center, \\ Columbus, $\mathrm{OH}$
}

\section{TO THE EDITORS:}

We thank Saluja et al. for their interest in our study ${ }^{1}$ and for their comments. Dr. Saluja et al. $^{2}$ bring up some interesting points. It is important to note that our study was a retrospective analysis of all patients who underwent surgical resection for primary gastric gastrointestinal stromal tumors (GISTs) at seven major institutions in the US and Canada over a 15 year time period (1998-2012). Indications for minimally invasive surgery (MIS) undoubtedly changed over the study period as familiarity and experience with MIS techniques improved. Additionally, there was substantial variation in the use of MIS by institution. In our study, the use of MIS was an individualized decision made by the treating surgeon based on tumor and patient characteristics, as well as surgical experience with MIS. The propensity match was completed to minimize this potential selection bias.

Our study was not a randomized controlled trial, therefore rather than completing an intention-to-treat analysis, we classified patients who underwent conversion to an open resection in the open group to allow for direct comparison of the type of resection actually received (MIS or open). An intention-to-treat sensitivity analysis was performed on the entire cohort with the conversion patients analyzed in the MIS group and no substantial changes were noted in the short- or long-term outcomes in either group. The results of this sensitivity analysis are described in our paper. ${ }^{1}$

\section{(C) Society of Surgical Oncology 2017}

First Received: 28 October 2017;

Published Online: 10 November 2017

D. A. Bischof, MD, MHS, FRCSC, FACS

e-mail: danielle.bischof@ sinaihealthsystem.ca
Despite propensity matching, in our study significantly more patients underwent gastric wedge resection in the MIS group compared with the open group following the propensity match. We believe that the differences in morbidity, blood loss, and length of stay in our study were quite substantial and likely not solely attributable to the type of gastrectomy completed. Unfortunately, data on incisional hernia and adhesive obstruction in follow-up were not available.

We agree that in the absence of a randomized controlled trial comparing MIS with open resection for resection of gastric GISTs, the potential for selection bias exists. In our study, the propensity-matched analysis was completed to minimize this risk. The objective of our study was to define the utilization of MIS and to characterize short- and longterm outcomes among patients undergoing MIS versus open surgery for gastric GISTs. We demonstrated that MIS is a safe technique associated with excellent short- and long-term outcomes for gastric GISTs, particularly for smaller tumors amenable to gastric wedge resection. We agree that open surgery should be considered for very large tumors, tumors in difficult locations, or when the surgeon's experience with MIS resection is limited.

DISCLOSURES None.

\section{REFERENCE}

1. Bischof DA, Kim Y, Dodson R, Jimenez C, Behman R, Cocieru A, et al. Open versus minimally invasive resection of gastric GIST: a multi-institutional analysis of short and long term outcomes. Ann Surg Oncol. 2014;21:2941-48.

2. Saluja S, Godhi S, Sisodia K, Mishra P. Comment on: open versus minimally invasive resection of gastric GIST: a multi-institutional analysis of short and long term outcomes. Ann Surg Oncol. 2017. https://doi.org/10.1245/s10434-017-6223-6. 